\title{
Prevalência de ortorexia nervosa em graduandos universitários da área de saúde:
}

\section{Uma revisão descritiva}

\author{
The prevalence of ortorexia nervosa among university healthy students: A descriptive review \\ Prevalencia de ortorexia nerviosa en estudiantes universitarios de salud: Revisión descriptiva
}

Recebido: 15/06/2021 | Revisado: 24/06/2021 | Aceito: 29/06/2021 | Publicado: 12/07/2021

\author{
Vitor Coêlho Silva Brandão \\ ORCID: https://orcid.org/0000-0002-2261-8296 \\ Universidade Federal de Pernambuco, Brasil \\ E-mail: vitorcsb@hotmail.com \\ Wanessa de Souza Xavier \\ ORCID: https://orcid.org/0000-0002-6868-0757 \\ Universidade Federal de Pernambuco, Brasil \\ E-mail:wanessa182@hotmail.com \\ Carmem Lygia Burgos Ambrósio \\ ORCID: https://orcid.org/0000-0002-5905-8077 \\ Universidade Federal de Pernambuco, Brasil \\ E-mail: carmem.ambrosio@ufpe.br \\ Raquel Araújo de Santana \\ ORCID: https://orcid.org/0000-0003-2768-015X \\ Universidade Federal de Pernambuco, Brasil \\ E-mail: raquela.santana@ufpe.br
}

\begin{abstract}
Resumo
A expressão Ortorexia Nervosa $(\mathrm{ON})$ está relacionada à "orthos" sendo indicativo de correto e "o-rexis" de apetite, sendo definida como uma obsessão por alimentação saudável. O público mais acometido é o de adolescente e adultos jovens do sexo feminino, e apresenta como consequência diversos prejuízos sociais e psicológicos. O objetivo do estudo foi descrever a prevalência de comportamentos com tendência a $\mathrm{ON}$ em graduandos universitários da área de saúde através de uma revisão descritiva. A pesquisa realizou-se nas bases PubMed, Scielo e Bireme por meio dos termos combinados (ortorexia nervosa) AND (graduandos universitários) OR (comportamento alimentar); (transtorno alimentar) AND (graduandos universitários) OR (comportamento alimentar); bem como seus respectivos termos em inglês. A busca resultou em 119 publicações e após a triagem, 11 estudos foram selecionados. Em relação às publicações foram extraídos os seguintes dados: título do estudo, ano de publicação, autores, objetivo, amostra e principais resultados. A maioria dos estudos selecionados abordou alunos do curso de Nutrição, porquanto acredita-se que eles constituem um grupo de risco em virtude do conhecimento sobre alimentação saudável, característica não observada em outro subgrupo populacional, e utilizou o questionário ORTO-15 para levantamento dos dados sobre alimentação. O percentual médio da presença de comportamentos de risco para ON foi aproximadamente $85,31 \%$. Os resultados demonstraram uma maior prevalência de comportamentos de risco entre mulheres. Observou-se, portanto, que os estudantes apresentaram elevada frequência de comportamentos de risco para o desenvolvimento da ON, tornando os mesmos suscetíveis as consequências sociais, psicológicas e de saúde motivadas por esse distúrbio.

Palavras-chave: Ortorexia nervosa; Transtornos alimentares; Graduandos universitários; Comportamento alimentar.
\end{abstract}

\begin{abstract}
The expression "Orthorexia Nervosa" (ON) is related to "orthos", an indicative of correct, and "o-rexis", of appetite, being defined like an obsession with healthy eating habit. The most affected public is the adolescents and young female adults, which presents as consequences of ON several social and psychological damages. The aim of this study was to describe the prevalence of tendency to $\mathrm{ON}$ in healthy university students through a descriptive review. The research was carried out on the bases Pubmed, Scielo, Bireme using the key words (orthorexia nervosa) AND (university students) OR (eating behavior); (eating disorder) AND (university students) OR (eating behavior); The Search resulted in 119 publications and after screening, 11 studies were selected. Regarding the publications where the following data were extracted: title of the study, year of publication, authors, objective, sample and main results. Most of the selected studies addressed students in Nutrition course, as they are believed to be a risk group due to their knowledge relative to healthy eating, a characteristic not observed in others populations subgroups, and used the ORTO-15 questionnaire to collect data about food. The average percentage of presence of risk behaviors for ON was approximately $85,31 \%$. The results showed a higher prevalence of risk behaviors among women. It was observed,
\end{abstract}


therefore, that students showed a high frequency of risk behaviors for the development of ON, making them susceptible to the social, psychological and health consequences motivated by this disorder.

Keywords: Orthorexia nervosa; Eating disorders; University students; Eating behavior.

\begin{abstract}
Resumen
Ortorexia Nervosa $(\mathrm{ON})$ se relaciona con que "ortos" sea indicativo de correcto y "o-rexis" del apetito, definiéndo-se como una obsesión por la alimentación saludable. El público más afectado son los adolescentes y jóvenes adultas, y como consecuencia presenta varios daños sociales y psicológicos. El objetivo del estudio fue describir la prevalencia de ON en estudiantes universitarios del campo de la salud a través de una revisión descriptiva. La investigación se llevó a cabo sobre las bases PubMed, Scielo, Bireme utilizando los términos combinados (ortorexia nerviosa) Y (estudiantes universitarios) OR (comportamento alimentario); (trastorno alimentario) Y (estudiantes universitarios) $\mathrm{O}$ (comportamiento alimentario). La búsqueda dio como resultado 119 publicaciones y, después de la selección, se seleccionaron 11 estudios. En relación a las publicaciones, se extrajeron los siguientes datos: título del estudio, año de publicación, autores, objetivo, muestra y principales resultados. La mayoría de los estudios seleccionados se dirigieron a estudiantes del curso de Nutrición, por considerarlos un grupo de riesgo por el conocimiento sobre alimentación saludable, característica no observada en otro subgrupo poblacional, y utilizaron el cuestionario ORTO15 para recolectar datos sobre alimentación. El porcentaje medio de presencia de conductas de riesgo para ON fue de aproximadamente $85,31 \%$. Los resultados mostraron una mayor prevalencia de conductas de riesgo entre las mujeres. Se observó, por tanto, que los estudiantes mostraron una alta frecuencia de conductas de riesgo para el desarrollo de la ON, haciéndolos susceptibles a las consecuencias sociales, psicológicas y de salud motivadas por este trastorno.

Palabras clave: Ortorexia nerviosa; Transtorno alimentario; Estudiantes universitarios; Comportamiento alimentario.
\end{abstract}

\title{
1. Introdução
}

As sociedades contemporâneas têm despertado um interesse cada vez maior pelos os fatores que afetam a saúde e longevidade humana, bem como um padrão de corpo arquétipo idealizado como belo e saudável e a qualidade da alimentação para atingir tais objetivos. As mudanças de comportamento social no ato de se alimentar, somadas a representação do corpo como objeto de identificação social, preconizado pelas mídias sociais, vem elevando o número de pessoas que apresentam algum tipo de disfunção relacionada à alimentação (Aparicio-Martinez et al., 2019). Nesse contexto, novos transtornos alimentares têm sido relatados, entre eles uma desordem alimentar relativamente recente é a Ortorexia Nervosa (ON), descrita pela primeira vez em 1997, pelo médico americano Steven Bratman, após o auto relato de ter sofrido de suas características.

A expressão ortorexia nervosa está correlacionada as palavras gregas “orthos" sendo indicativo de preciso ou correto e "o-rexis" de apetite, portanto pode ser definido como um comportamento obsessivo por uma nutrição adequada (Varga, Thege, Dukay-Szabó, Túry, \& van Furth, 2014) ou obsessão por alimentação saudável com relação a qualidade e a pureza dos alimentos (Rodrigues, de Oliveira, Garcia \& de Omena Messias, 2017). Um ponto marcante da ON e que a diferencia dos outros distúrbios é a falta de obsessão por um corpo esbelto ou pela quantidade de alimentos ingeridos, sendo sua característica principal a obsessão pela qualidade do que vai ser consumido (de Almeida Luna \& Belmonte 2016; Plitcha \& JezewskaZychowicz, 2019).

Segundo Douma, Valente e Syurina (2021), a ON é um transtorno desenvolvido ao longo do tempo e está relacionado a influência biológica, psicológica e social, ou seja, não é algo que se inicia de forma repentina. Por outro lado, é um distúrbio com difícil diagnóstico e busca por tratamento, pois as pessoas acometidas acreditam que seu comportamento alimentar está correto e deve ser seguido por todos (Plitcha \& Jezewska-Zychowicz, 2020). Em determinadas situações, a dieta se torna a parte mais importante da vida da pessoa, e a alimentação se torna tão restrita em variedade e calorias, que afeta a saúde do indivíduo acometido pelo transtorno (Coelho, Troglio, Hammes, Galvão \& Cyrino, 2016).

O público mais acometido pelos distúrbios alimentares é o de adolescente e adultos jovens, do sexo feminino, por possuírem comportamentos subclínicos precursores (Córdas, 2004; De Marchi \& Baratto, I. 2018). Tendo como consequência diversos prejuízos sociais e psicológicos uma vez que há o isolamento social das pessoas com características ortoréxicas pelo 
sentimento de superioridade alimentar e a necessidade de impor seu comportamento alimentar aos membros do seu ciclo social (Pontes \& Montganer, 2014; Vilarinho, 2020).

Além disso, há o aumento na prevalência desses distúrbios entre a população estudantil, elevando o número de estudos que buscam relatar as consequências dos mesmos para a vida educacional e social das pessoas acometidas por eles. (Santos, 2017). Observa-se na literatura uma alta prevalência de transtornos de imagem corporal em estudantes da área da saúde, como medicina, nutrição, educação física e atletas, nos quais os aspectos da forma física são relevantes (de Almeida Luna \& Belmonte 2016; Vital, Silva, Garcia \& de Omena Messias, 2017).

Dessa forma, devido à relevância do tema, não somente para os profissionais da saúde como para a população geral, percebe-se então a importância de um melhor entendimento acerca dos fatores de risco associados à ortorexia nervosa, da sua prevalência em graduandos da área de saúde, e consequentemente, da sua presença moldando a vida desses graduandos universitários. Assim, o objetivo desse estudo é descrever, através de uma revisão descritiva, a prevalência de comportamentos de tendência a $\mathrm{ON}$ em graduandos universitários da área de saúde.

\section{Metodologia}

Tratou-se de revisão descritiva de artigos científicos para obtenção dos conhecimentos produzidos acerca do tema ortorexia, de modo ordenado e sintético dos desfechos mais comuns.

\subsection{Estratégia de busca dos estudos}

Para a definição das palavras-chave foi realizada uma consulta no Mesh (Medical Subject Headings) e no DeCS (Descritores em Ciências da Saúde). Foram utilizados os termos "ortorexia nervosa" e "transtornos alimentares" como descritores primários e os termos "graduandos universitários" e "comportamento alimentar" como descritores secundários.

A busca eletrônica dos artigos que integraram a pesquisa foi realizada nos meses de janeiro e fevereiro de 2019 nas bases de dados U.S. National Library of Medicine (PubMed), Scientific Electronic Library Online (Scielo) e Biblioteca Virtual em Saúde (Bireme) por disponibilizarem grande conteúdo de pesquisas.

Nas bases de dados as buscas foram realizadas da seguinte maneira: (ortorexia nervosa) AND (graduandos universitários) OR (comportamento alimentar); (transtorno alimentar) AND (graduandos universitários) OR (comportamento alimentar); bem como seus respectivos termos em inglês.

\subsection{Critérios de elegibilidade dos estudos}

Foram considerados para análise somente artigos originais, que investigassem a prevalência de comportamentos com tendência a ON em graduandos universitários, artigos publicados em língua portuguesa, inglesa ou espanhola, estudos com abordagem quantitativa, qualitativa ou mista, publicados a partir do ano de 2009 e com textos completos disponíveis na íntegra pelo meio on-line. Foram excluídos artigos de revisão, resumos de congresso, editoriais e cartas, por se tratarem de dados não originais ou incompletos.

\subsection{Seleção dos estudos e extração de dados}

Após a busca dos artigos, iniciou-se a seleção dos estudos por meio da análise dos títulos. Posteriormente, foram analisados os resumos e na terceira etapa foi realizada a análise do texto na íntegra dos artigos selecionados nas etapas anteriores. 
Para a extração dos dados, foi construído um formulário específico, onde foram registradas informações relacionadas ao título do estudo, autores, ano de publicação, objetivo, amostra e principais resultados verificados.

\section{Resultados}

A busca resultou em 119 registros. Após aplicação dos critérios de elegibilidade foram selecionados 38 artigos para leitura do resumo. Nesta etapa foram excluídos outros 27 estudos por não acrescentarem informações relevantes ao estudo. Fizeram parte da revisão final 11 publicações, conforme fluxograma abaixo (Figura 1).

Figura 1: Processo de seleção dos estudos sobre Ortorexia Nervosa em graduandos universitários.
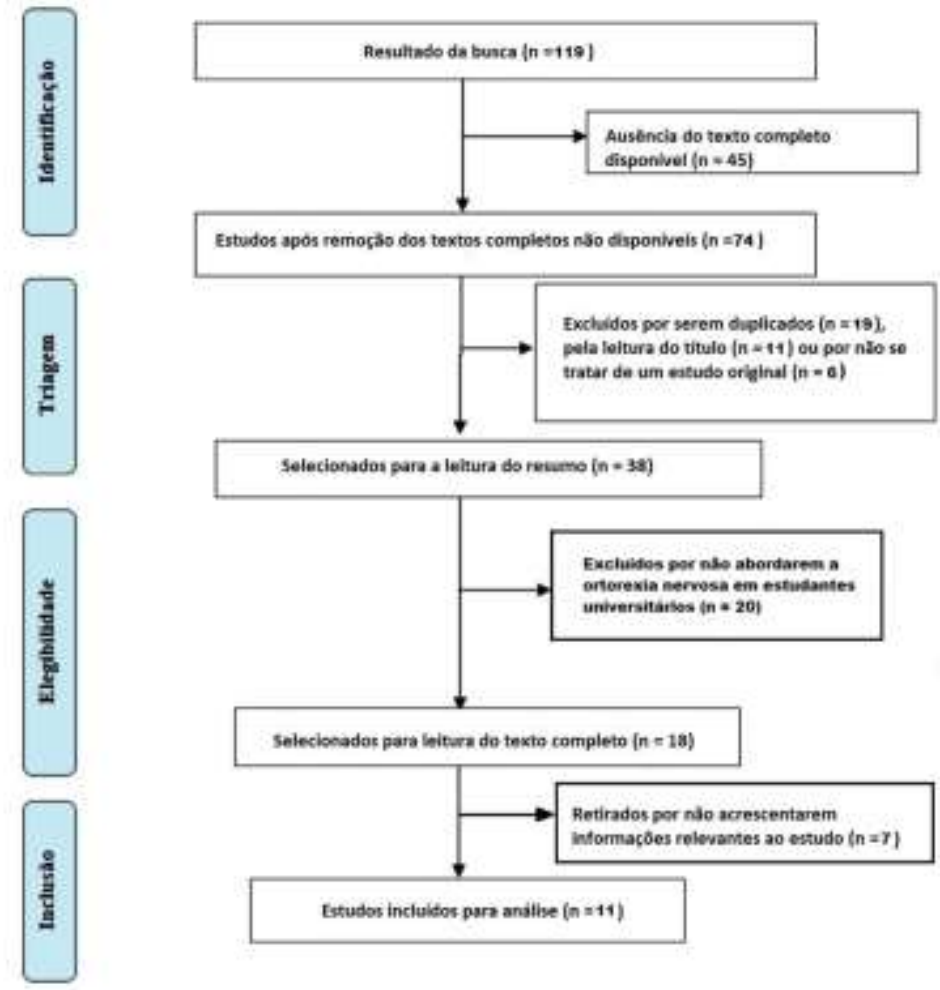

Fonte: Autores.

Os estudos indicam altas taxas de comportamentos com tendência a ortorexia nervosa nos graduandos universitários, principalmente do curso de Nutrição, sugerindo que as principais características da ortorexia se desenvolvem significativamente em pessoas que buscam um maior conhecimento sobre alimentação saudável e adequada em todos os aspectos da vida. (Tabela 1).

Entretanto, os resultados do estudo de Vital et al. (2017) revelam que comportamentos que podem levar a ON estão presentes também entre os graduandos de Educação Física, que demonstram interesse em melhorar a alimentação para obtenção de um desempenho físico ideal. (Tabela 1).

Apenas o estudo de Lopes e Kirsten (2009), não encontrou indícios de comportamento de ortorexia nas mulheres jovens estudantes de graduação dos cursos de Nutrição, Farmácia e Enfermagem. (Tabela 1). 
Tabela 1. Descrição da amostra, objetivos e principais resultados dos estudos sobre ON em graduandos universitários.

\section{No TÍTULO} DO AUTORES

ANO DE OBJETIVO

1 Comportamento de Souza risco para ortorexia Rodrigues nervosa em estudantes de

nutrição

Ortorexia nervosa em Penaforte et 2017 estudantes de al.

nutrição:

associações com o

estado nutricional,

satisfação corporal e

período cursado

3 Prevalência de

ortorexia nervosa em acadêmicos do

curso de nutrição em uma instituição

de ensino superior no sudoeste do Paraná

4 Comportamentos de ortorexia de Nervosa em mulheres

jovens

Risco de ortorexia Rodrigues et 2017 nervosa e o al. comportamento

alimentar

estudantes do Sul. al.

De Marchi e 2018

Baratto

Identificar comportamento de risco para o desenvolvimento de ortorexia nervosa em estudantes de nutrição.

Identificar a prevalência de comportamentos com tendência a ortorexia nervosa $(\mathrm{ON})$ e suas associações com o estado nutricional, satisfação corporal e período cursado em estudantes de nutrição.

Verificar a presença de ortorexia nervosa em estudantes do curso de nutrição de uma instituição de ensino superior no sudoeste do Paraná.

$\begin{array}{lll}\text { Lopes } & \text { e } & 2009 \\ \text { Kirsten } & & \end{array}$

\section{AMOSTRA}

$\mathbf{N}(\%)$

$150(100 \% \mathrm{~F})$

No que se refere à ortorexia nervosa, foi observado que $88,7 \%$ das alunas que participaram do estudo apresentavam risco de desenvolver comportamento ortoréxico. Porém ao relacionar o mesmo com a série cursada, o estado nutricional e o distúrbio da imagem corporal, verificou-se que não houve associação significativa entre as variáveis.

$141(9,2 \% \mathrm{M}$ e Foram identificados em 87,2\% dos estudantes 90,8\% F) avaliados, comportamentos alimentares com tendência a ON. Os mesmos, apresentaram maior incidência de insatisfação corporal e excesso de peso. Porém, não foi observada associação entre a ON e o período cursado.

$82(6 \% \mathrm{M}$ e De acordo com os dados $80 \%$ apresentaram $94 \% \mathrm{~F}$ ) prevalência para a ortorexia. Das variáveis, o IMC mostrou que quanto mais baixo o seu valor, maior a probabilidade ao comportamento. Com relação ao gênero, todos os participantes do sexo masculino apresentaram comportamento ortoréxico, os participantes do sexo feminino somaram $92 \%$ do percentual.

Verificar comportamentos de $200(100 \%$ F)

Ortorexia Nervosa em estudantes do *Nutrição, sexo feminino de Cursos da Área da Saúde de uma faculdade particular do centro do estado do Rio Grande

Avaliar o comportamento de risco para desenvolvimento de ortorexia nervosa e o comportamento alimentar de estudantes de bacharelado em nutrição. enfermagem e saúde, pois a maioria das respostas não foram farmácia caracterizadas como distúrbios de comportamentos adversos à alimentação normal.

113 (16\% M e Constatou-se que 94,69\% dos discentes apresentaram $84 \%$ F) traços de ortorexia, sendo o sexo masculino com maior prevalência. Não houve associação estatística significativa entre os grupos dos diferentes semestres analisados. Também foi observada inadequação de 


\section{nutrição}

6 Risco

desenvolvimento de

ortorexia nervosa e o

comportamento

Alimentar

de

graduandos

universitários

7 Comportamento de Anjos

risco para

desenvolvimento de

ortorexia nervosa em

estudantes de

nutrição do centro

universitário de

Brasília

$8 \quad$ Ortorexia nervosa em Santos

estudantes de

nutrição: o vício de

comer saudável.

Risco de Moura

desenvolvimento de ortorexia nervosa em estudantes de nutrição do sexo feminino de uma universidade no município de Palhoça (SC)

10 Avaliação de Tezza et al. sintomas de ortorexia nervosa em estudantes do

curso de Nutrição em uma universidade do
Avaliar \begin{tabular}{llllll} 
Avaliar & \multicolumn{2}{c}{ risco } & para & o & 40 \\
desenvolvimento & de & ortorexia & $37,5 \% \mathrm{~F})$ \\
nervosa e & o & comportamento & $*$ Ed. Física \\
alimentar & de & graduandos &
\end{tabular} universitários.

Identificar características que $101(12,8 \% \mathrm{M} \mathrm{e}$ propiciar o $87,1 \% \mathrm{~F}$

desenvolvimento de comportamento ortoréxico nervoso em universitários do curso de nutrição do Centro Universitário de Brasília. Avaliar a prevalência de ortorexia
nervosa em estudantes de nutrição.

$191(12 \% \mathrm{M} \mathrm{e}$ $88 \% \mathrm{~F})$

Verificar risco para Ortorexia Nervosa (ON) em estudantes de Nutrição do sexo feminino e sua associação com o estado nutricional, com a satisfação com imagem corporal (SIC) e com semestre cursado.

Avaliar sintomas de Ortorexia $291(100 \%$ F) Nervosa em estudantes do sexo *97 Nutrição feminino, acadêmicas do curso de *194 Outros Nutrição de uma faculdade cursos particular do sul de Santa Catarina.

$89(100 \% \mathrm{~F})$ hábitos alimentares.

relação à ortorexia nervosa, constatou-se que $82,5 \%$ dos indivíduos apresentaram comportamento de risco para desenvolvê-la, prevalentemente no sexo masculino (88\%). Com relação à série do curso, os alunos do primeiro período apresentaram maior risco quando comparados ao sétimo período. Quanto ao comportamento alimentar, escolhas saudáveis predominaram.

Foi relatada uma prevalência de $88,1 \%$ dos estudantes com indicativos de ON, e observada sua ligação aos primeiros semestres do curso, predomínio de classificação nutricional de peso normal, e de encontro à taxa de insatisfação corporal que foi de $75,2 \%$.

De acordo com as respostas do ORTO-15 foi analisado que tanto no grupo feminino, quanto no masculino a quantidade de estudantes com tendência a ter ortorexia nervosa é relevante, sendo no feminino $82,7 \%$ e no masculino $100 \%$.

Dentre as avaliadas $84,3 \%$ apresentavam risco para ON. Apenas 29,2\% das estudantes estavam satisfeitas com a imagem corporal, $\mathrm{O}$ risco de $\mathrm{ON}$ foi $84 \%$ menor para as estudantes das fases mais adiantadas do curso e $81 \%$ menor para as estudantes satisfeitas com a imagem corporal.

Não foram verificadas diferenças na média de IMC entre os grupos caso e controle. O grupo caso, apresentou uma chance cerca de cinco vezes maior de achar que mantêm sua alimentação totalmente controlada e saudável do que o controle. No grupo caso também foi verificada uma chance quase dua 
Research, Society and Development, v. 10, n. 8, e30110817207, 2021

(CC BY 4.0) | ISSN 2525-3409 | DOI: http://dx.doi.org/10.33448/rsd-v10i8.17207

sul do país

11 Verificação do estado Cruz et al. nutricional de estudantes do curso de nutrição das

faculdades integradas

de Bauru- SP com

enfoque na ortorexia vezes maior de apresentarem o escore indicativo de ON no questionário ORTO-15, quando comparadas ao grupo controle.

Verificar por meio de uma $24(12,5 \% \mathrm{M}$ e Observou-se que $71 \%$ dos estudantes encontram-se anamnese alimentar o perfil de $87,5 \%$ F) eutróficos, $80 \%$ com circunferência abdominal dentro estudantes de nutrição, como também a tendência ao quadro de ortorexia nervosa e distorção da imagem corporal, utilizando os questionários Body Shape Questionary (BSQ) e Orto-15. eutróficos, $80 \%$ com circunferência abdominal dentro
do esperado, porém, ainda assim, $71 \%$ dos participantes apresentaram tendência ao quadro de ortorexia.

Legenda: $\mathrm{F}=$ feminina; $\mathrm{M}=$ masculina; $\mathrm{ON}$ = ortorexia nervosa. Fonte: Autores 


\section{Discussão}

De acordo com a Organização Mundial da Saúde (OMS), a saúde deixa de ser considerada apenas a ausência de doença e amplia seu conceito ao completo bem-estar físico, mental e social. Dessa forma, apesar das pessoas com comportamentos ortoréxicos serem consideradas mais saudáveis (Plitcha \& Jezewska-Zychowicz, 2020; Souza \& Rodrigues, 2014) há um problema a respeito do convívio social, visto que as pessoas com ON tendem a fazer as refeições sozinhas e evitam se alimentar fora de casa, por não saberem a procedência daquele alimento, causando isolamento do convívio social (Bayão \& Damous, 2020).

Obeid, Hallit, Akel e Brytek-Matera (2021) relatam que desregulação emocional é um forte fator preditor de ON, demonstrando que falta de controle emocional pode estar relacionado ao desenvolvimento do comportamento ortoréxico. Dessa forma, graduandos universitários se tornam um grupo de risco (Santos, 2017), visto que estão inseridos em ambientes de pressão e dificuldades psicológicas. Além disso, tal problema é fomentado no público universitários da área de saúde, como relatou Aidar et al. (2020), devido à preocupação com as calorias ingeridas, ansiedade, insatisfação corporal, entre outros fatores associados que podem provocar desregulação mental.

Entre os 11 estudos selecionados para o desenvolvimento deste trabalho, apenas o de Vital et al. (2017), não envolveu estudantes de nutrição, sendo realizado com estudantes de educação física. Isso demonstra uma preocupação relevante com graduandos do curso de nutrição por acreditar-se que eles constituem um grupo de risco para o desenvolvimento da ON. Essa atenção pode ser justificada, de acordo com Penaforte, Barroso, Araújo e Japur, (2018), pela pressão social inerente à profissão em relação ao peso, aparência física e o hábito alimentar. Assim, se tornam mais propícios ao desenvolvimento de distúrbios alimentares.

Já o estudo de Tezza, Iser, Turatti e Warmling (2018), foi o único a apresentar uma comparação entre estudantes do curso de Nutrição, (grupo caso), com estudantes de graduação de quaisquer outros cursos com mesmo nível sociocultural e econômico (constituindo o grupo controle), demonstrando que o grupo caso apresentou uma probabilidade cinco vezes maior de acreditar que mantêm sua alimentação totalmente controlada e saudável, e uma chance quase duas vezes maior de apresentarem o escore indicativo de ON no questionário orto-15, quando comparadas ao grupo controle.

$\mathrm{O}$ único estudo que não encontrou características de risco para o desenvolvimento da ON nos estudantes foi o de Lopes e Kirsten (2009), que envolviam os cursos de nutrição, enfermagem e farmácia. Por se tratar de um estudo anterior à adaptação e tradução para o português do questionário ORTO-15, acredita-se que este resultado esteja relacionado a utilização de uma metodologia não ajustada para a melhor identificação de comportamentos ortoréxicos. Todos os outros estudos analisados utilizaram o ORTO-15 na sua metodologia.

Com relação ao gênero mais acometido, os resultados demostraram uma maior prevalência de comportamentos de risco para ON entre mulheres, apesar do sexo masculino ter atingido a totalidade da amostra desse gênero em alguns estudos incluídos na revisão (De Marchi \& Baratto, 2018; Rodrigues et al., 2017; Vital et al., 2017; Anjos \& Solomon, 2018; Santos, 2017). Foi identificado nesse gênero, uma preocupação maior com relação as fases de escolha, aquisição e preparo dos seus alimentos. O sexo feminino teve maior predominância nos estudos, e aparentemente maior risco no desenvolvimento do comportamento ortoréxico.

Quando associada ao Índice de Massa Corporal (IMC), a maioria dos estudos não encontraram relação entre as variáveis. Já os trabalhos de Souza e Rodrigues (2014) e Penaforte et al. (2017), relataram uma frequência maior em indivíduos que apresentavam excesso de peso ou obesidade. Em contrapartida, De Marchi e Baratto (2018), encontrou uma associação inversa dessas variáveis, sendo a $\mathrm{ON}$ mais presente em pessoas que apresentavam um IMC menor. 
Os autores que compararam os comportamentos de risco para a ortorexia com a satisfação corporal, constataram uma relação significativa entre aqueles com maior frequência de insatisfação e os estudantes com tendência para a ON. O estudo de Souza e Rodrigues (2014), não encontrou correlação entre comportamento ortoréxico e distorção da imagem corporal, visto que a ortorexia não representa um transtorno de imagem onde há uma busca por um corpo ideal, mas sim por uma melhora da saúde e qualidade de vida.

Ao relacionar os comportamentos ortoréxicos e o período do curso em que os estudantes estavam no momento das pesquisas, a maior parte dos autores não observaram uma associação significativa (Souza \& Rodrigues, 2014; Penaforte et al., 2017; De Marchi \& Baratto, 2018; Rodrigues et al., 2017; Santos, 2017). Entretanto, os estudos de Vital et al. (2017) e Anjos (2018), relataram uma maior prevalência de comportamentos de risco, nos períodos iniciais do curso.

\section{Considerações Finais}

Por meio da análise dos estudos incluídos na revisão constata-se que os universitários apresentaram elevada frequência de comportamentos de risco para o desenvolvimento da ortorexia nervosa, tornando os estudantes suscetíveis as consequências sociais, psicológicas e de saúde motivadas por esse distúrbio.

Como limitação desta revisão, constata-se que o ORTO-15 utilizado nos estudos elencados não se destina ao diagnóstico da ortorexia nervosa, mas de identificação do risco de desenvolvimento do transtorno. Desta forma, seria inadequado estabelecer a prevalência de ortorexia em estudantes da saúde, podendo apenas sugerir um risco em torno de $80 \%$ de ON nesse público.

Os hábitos inadequados foram frequentes entre homens, visto que estes apresentaram uma preocupação maior sobre a sua alimentação. Essas condutas também foram observadas entre os indivíduos que apresentaram IMC inadequado, reforçando a ideia de que a ortorexia não busca a magreza, mas sim o peso ideal para uma melhor condição de saúde e qualidade de vida. Fortalecendo, observou-se também que há uma ligação entre insatisfação com a imagem corporal e ortorexia. Por fim, não foi encontrada uma associação significativa quando se analisou o período em que os estudantes se apresentavam.

Embora a ortorexia nervosa ainda não seja oficialmente reconhecida como um Transtorno Alimentar ou um Transtorno Obsessivo, o grande obstáculo encontrado em relação a esta doença é a linha tênue existente entre alimentação saudável e o comportamento de obsessão patológica do paciente pelo que ele acredita ser "alimentação saudável", sem a devida orientação de profissionais da área de nutrição.

Diante da importância desta problemática, sugere-se que sejam realizados novos estudos sobre a ON, em graduandos universitários da área de saúde a fim de se compreender melhor as causas que conduzem a este desfecho e, a partir daí, procurar estratégias almejando à prevenção e o tratamento deste distúrbio, uma vez que o mesmo influencia de forma direta e negativa na vida social dos indivíduos.

\section{Referências}

Aidar, M. D. O. I., Freitas, R. B. D., Bastos, G. C. F. C., Brasileiro, A. A., Silva, A. M. T. C., \& Almeida, R. J. D. (2020). Fatores Associados à Suscetibilidade para o Desenvolvimento de Transtornos Alimentares em Estudantes Internos de um Curso de Medicina. Revista Brasileira de Educação Médica, 44(3).

Anjos, L. P. D., \& Salomon, A. L. R. (2018). Comportamento de risco para desenvolvimento de ortorexia nervosa em estudantes de nutrição do Centro Universitário de Brasília. Monografia (Graduação), Faculdade de Ciências da Educação e Saúde, Centro Universitário de Brasília, Brasília, DF, Brasil.

Aparicio-Martinez, P., Perea-Moreno, A. J., Martinez-Jimenez, M. P., Redel-Macías, M. D., Pagliari, C., \& Vaquero-Abellan, M. (2019). Social media, thinideal, body dissatisfaction and disordered eating attitudes: An exploratory analysis. International journal of environmental research and public health, 16(21), 4177. 
Bayão, B., \& Damous, I. (2020). Ortorexia: problematizando a busca pela alimentação saudável na contemporaneidade. ECOS-Estudos Contemporâneos da Subjetividade, 10(2), 173-183.

Coelho, G. C., Troglio, G. M., Hammes, L., Galvão, T. D., \& Cyrino, L. A. R. (2016). As consequências físicas, psíquicas e sociais em indivíduos com ortorexia nervosa. RBONE-Revista Brasileira de Obesidade, Nutrição e Emagrecimento, 10(57), 160-168.

Cordás, T. A. (2004). Transtornos alimentares: classificação e diagnóstico. Rev. psiquiatr. clín, 154-157.

Cruz, R. T., Souza, C. T., Francisqueti, F. V., \& Souza, D. T. (2018). Verificação do estado nutricional de estudantes do curso de Nutrição das Faculdades Integradas de Bauru-SP com enfoque na ortorexia. RBONE-Revista Brasileira De Obesidade, Nutrição E Emagrecimento, 12(76), 1119-1128.

de Almeida Luna, C., \& Belmonte, T. D. S. A. (2016). Ortorexia nervosa: um desafio para o nutrólogo. International Journal of Nutrology, 9(01), 128-139.

De Marchi, P., \& Baratto, I. (2018). Prevalência de ortorexia nervosa em acadêmicos do curso de Nutrição em uma Instituição de Ensino Superior no sudoeste do Paraná. RBONE-Revista Brasileira De Obesidade, Nutrição E Emagrecimento, 12(74), 699-706.

Douma, E. R., Valente, M., \& Syurina, E. V. (2021). Developmental pathway of orthorexia nervosa: Factors contributing to progression from healthy eating to excessive preoccupation with healthy eating. Experiences of Dutch health professionals. Appetite, 158, 105008.

Lopes, M. R., \& Kirsten, V. R. (2009). Comportamentos de ortorexia nervosa em mulheres jovens. Disciplinarum Scientia| Saúde, 10(1), 97-105.

Moura, P. C. (2018). Risco de desenvolvimento de ortorexia nervosa em estudantes de nutrição do sexo feminino de uma universidade no município de Palhoça (SC). Nutrição-Pedra Branca. Universidade do Sul de Santa Catarina, Palhoça, Santa Catarina (SC), Brasil.

Obeid, S., Hallit, S., Akel, M., \& Brytek-Matera, A. (2021). Orthorexia nervosa and its association with alexithymia, emotion dysregulation and disordered eating attitudes among Lebanese adults. Eating and Weight Disorders-Studies on Anorexia, Bulimia and Obesity, 1-10.

Penaforte, F. R., Barroso, S. M., Araújo, M. E., \& Japur, C. C. (2018). Ortorexia nervosa em estudantes de nutrição: associações com o estado nutricional, satisfação corporal e período cursado. Jornal Brasileiro de Psiquiatria, 67(1), 18-24.

Plichta, M., Jezewska-Zychowicz, M., \& Gębski, J. (2019). Orthorexic tendency in Polish students: exploring association with dietary patterns, body satisfaction and weight. Nutrients, 11(1), 100.

Plichta, M., \& Jezewska-Zychowicz, M. (2020). Orthorexic tendency and eating disorders symptoms in Polish students: Examining differences in eating behaviors. Nutrients, 12(1), 218.

Pontes, J. B., Montagner, M. I., \& Montagner, M. Â. (2014). Ortorexia nervosa: adaptação cultural do orto-15. Demetra: Alimentação, Nutrição \& Saúde, $9(2), 533-548$.

Rodrigues, B. C., de Oliveira, G. N. S., Garcia, E. I., \& de Omena Messias, C. M. B. (2017). Risco de ortorexia nervosa e o comportamento alimentar de estudantes de nutrição. Scientia plena, 13(7).

Santos, M. (2017). Padrão Alimentar Anormal em Estudantes Universitárias das Áreas de Nutrição, Enfermagem e Ciências Biológicas. Ciência ET Praxis, 1(01), 1-4. Recuperado de https://revista.uemg.br/index.php/praxys/article/view/2064.

Santos, K. M. D. A. (2017). Ortorexia nervosa em estudantes de nutrição: o vício de comer saudável. Artigo (Graduação), Faculdade de Ciências da Educação e Saúde, Centro Universitário de Brasília, Brasília, DF, Brasil.

Souza, Q. J. O. V. D., \& Rodrigues, A. M. (2014). Comportamento de risco para ortorexia nervosa em estudantes de nutrição. Jornal brasileiro de psiquiatria, 63(3), 200-204.

Tezza, M. Z., Iser, B. M., Turatti, C., Lin, J., \& Warmling, M. (2018). Avaliação de sintomas de ortorexia nervosa em estudantes do curso de Nutrição em uma universidade do sul do país. RELATOS DE CASOS, 62(2), 148-153.

Varga, M., Thege, B. K., Dukay-Szabó, S., Túry, F., \& van Furth, E. F. (2014). When eating healthy is not healthy: orthorexia nervosa and its measurement with the ORTO-15 in Hungary. BMC psychiatry, 14(1), 1-11.

Vilarinho, B. A. (2020). Contexto da ortorexia nervosa e o comportamento de risco em estudantes do curso de nutrição: uma revisão de literatura. Monografia (Graduação), Faculdade de Ciências da Educação e Saúde, Centro Universitário de Brasília, Brasília, DF, Brasil.

Vital, A. N. S., Silva, A. B. A., Garcia, E. I., \& de Omena Messias, C. M. B. (2017). Risco para desenvolvimento de ortorexia nervosa e o comportamento alimentar de graduandos universitários. Saúde e Pesquisa, 10(1), 83-89. 